\title{
Spinal Muscular Atrophy Type 3: A Case Report
}

\author{
MARIA KIBTIAR ${ }^{1}$, ROKSANA PARVIN ${ }^{1}$, MANIK KUMAR TALUKDER ${ }^{2}$, CHOUDHURY ALI KAWSER ${ }^{3}$
}

\begin{abstract}
Spinal muscular atrophy (SMA) type 3 is a relatively stable genetically determined chronic neuromuscular disorder caused by degeneration of motor neurons of spinal cord. Patients with type 3 SMA may gradually experience decline in muscle strength and motor function. However functional progression is difficult to document and mechanisms remain poorly understood. A five years old boy presented with proximal muscle weakness, generalized hypotonia, absent deep tendon reflexes and features of neuropathy and labeled as SMA type 3.
\end{abstract}

Key words: Spinal muscular atrophy (SMA), creatinine kinase, electromyography.

\section{Introduction:}

Spinal muscular atrophy (SMA) is a neurodegenerative disease with autosomal recessive inheritance. The incidence of this disease is 1:1000 births. ${ }^{1}$ The frequency of carriers (heterozygotes) is 1 in 40 to 60 people. $^{2}$ The disease is caused by homozygous mutation or deletion of the survivor motor neuron gene (SMN1), which is located in the telomere region of chromosome $5 q 13 .{ }^{1}$ Clinically SMA is classified into several types based on age of onset and degree of motor function achieved by the affected individual. ${ }^{3,4}$ Among them type 3 SMA patients have less severe symptoms and are able to walk and reach the major motor milestones but often loss the ability to walk over time as disease progresses. Because symptoms of type 3 SMA appears later in childhood, generally these patients are diagnosed later. ${ }^{3,5}$ Diagnosis is based on evidence of denervation of muscle both in electrophysiology and histopathology. ${ }^{6}$ There is no specific treatment of this disease. Medical follow up and palliative care both are important in entire life of patient's with SMA. Genetic counseling is an essential component for the families of these patients. ${ }^{1}$ SMA type 3 is an uncommon disease and our case presented only at age of 5 years. That is why we are reporting the case.

1. Resident, General Paediatrics, BSMMU, Dhaka

2. Associate Professor, Department of Paediatrics, BSMMU, Dhaka

3. Professor, Department of Paediatrics, BSMMU, Dhaka

Correspondence: Dr. Maria Kibtiar, Resident, Phase B, General

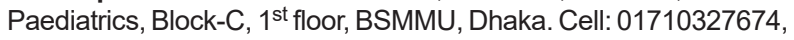
e-mail: mariabsmmu@yahoo.com

Received: 10/11/2018

\section{Case report:}

A 5 years old boy, born to healthy non consanguineous parents admitted at the department of Paediatrics, Bangabandhu Sheikh Mujib Medical University (BSMMU) with the complaints of progressive weakness with inability to sit and stand without assistance since 18 months of age. He also had history of frequent fall on walking for last 2 years. Moreover he had difficulty in combing hair and raising his hand above head. He was delayed in achieving motor skills as he sat with assistance at 12 months and walked after assisted standing at 20 months of age. But his vision, hearing and cognition were normal. There was no history of recurrent respiratory tract infection, sucking or swallowing difficulty and no features of hypothyroidism. His elder sister, who is 10 years old is suffering from same type of illness but in milder form since she started to stand. Now she can walk without any support but cannot sit or stand without assistance. On examination, the boy was well alert (Figure1). His higher mental function and cranial nerve examination were normal. There was no fasciculation, no evidence of muscle atrophy or hypertrophy (Figure 1 and 2). There was generalized hypotonia with reduced muscle power (3/4), mostly in proximal muscles of both upper and lower limbs and jerks were absent in all four limbs. So provisionally we diagnosed the case as myopathy. Investigation showed normal muscle enzymes (CPK, LDH, Aldolase and AST). Nerve conduction velocity (NCV) was normal (Figure 3). Electromyography (EMG) showed non inflammatory myopathy in lower limb muscles mostly in Quadriceps, tibialis anterior 


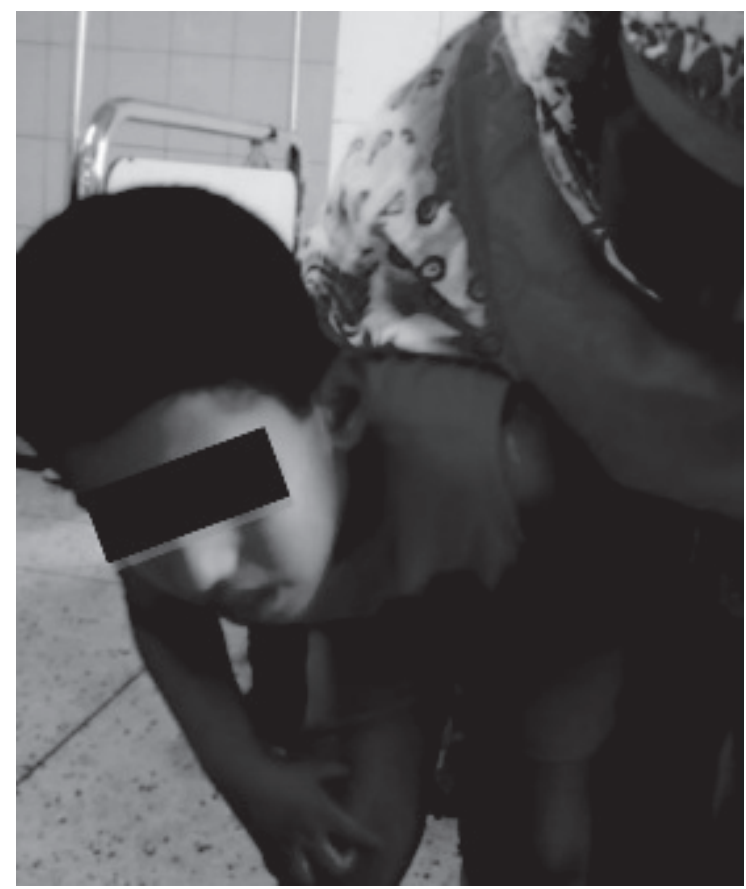

Fig.-1: Not able to stand or sit without assistance

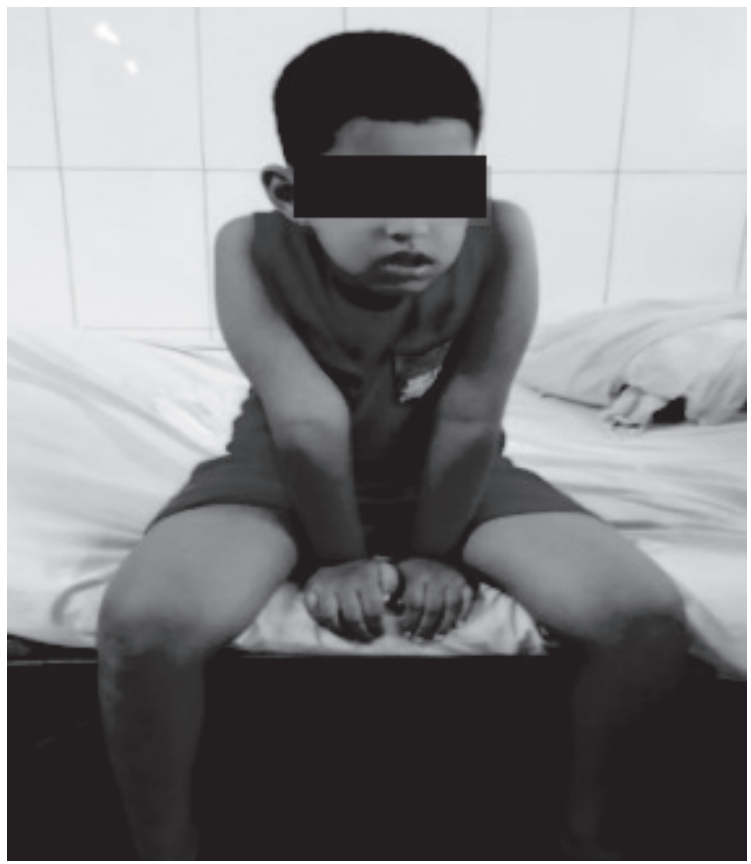

Fig.-2: Masked face with normal muscle bulk

and gluteus medius muscle (Figure 4) and also in biceps brachii (Figure 5) muscle of upper limb. Muscle biopsy reported small diameter muscle fibres with mild stromal fatty infiltration compatible with spinal muscular atrophy type 3 . So finally a diagnosis of spinal muscular atrophy type 3 was made.

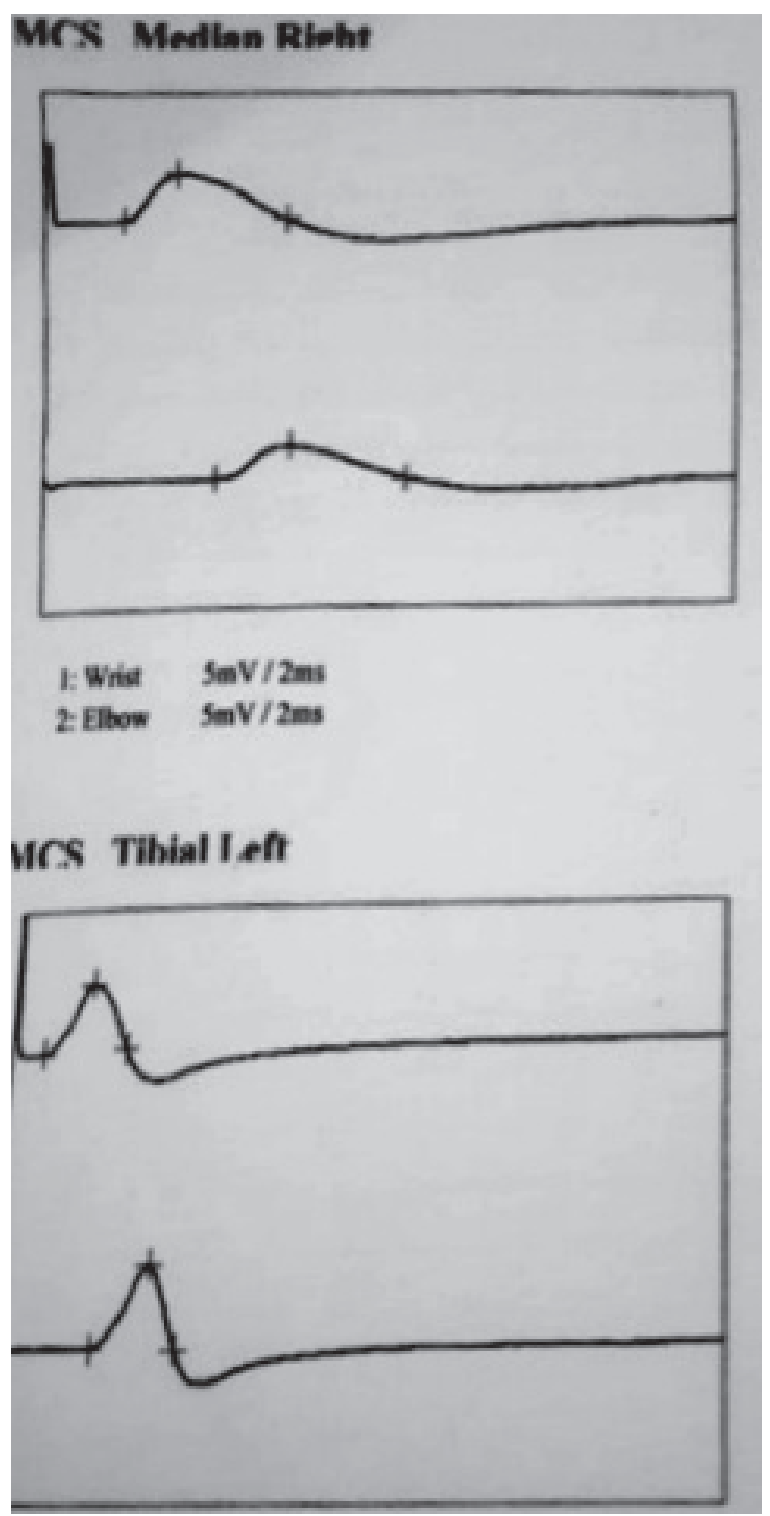

Figu.-3: Normal NCV

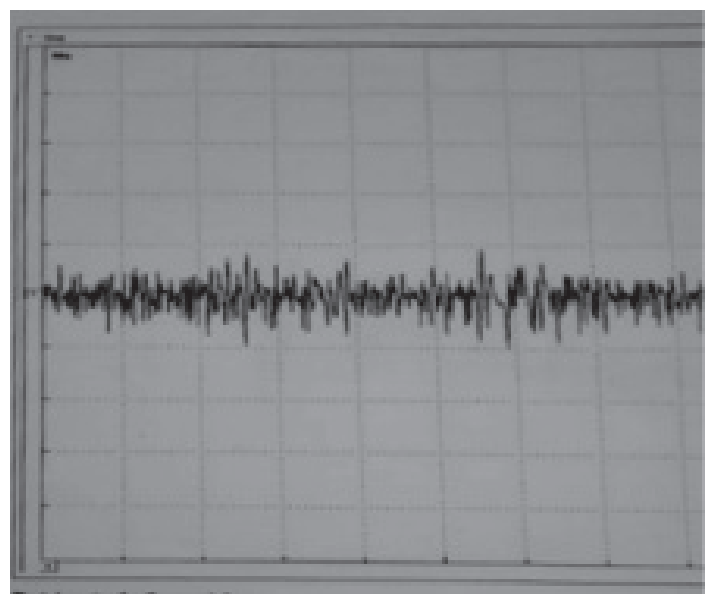

Fig.- 4: Myopathic changes of Gluteus medius muscle in EMG 


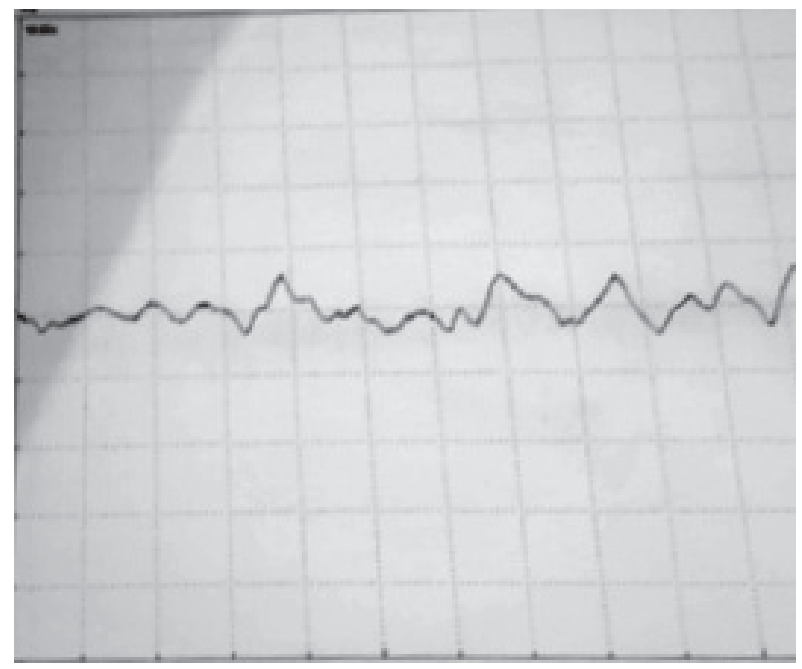

Fig.-5: Myopathic changes of biceps brachii muscle in $E M G$

\section{Discussion:}

Spinal muscular atrophy (SMA) refers to group of genetic disorders caused by degeneration of anterior horn cells and resultant muscle atrophy and weakness. ${ }^{7}$ Based on disease onset and severity, childhood onset SMA can be classified into 3 major types. Most severe form is Type 1 (Werdnig Hoffman) disease, with onset within first 6 month. A intermediate form, Type 2 (Dubowitz disease), with onset before 18 months and Type 3 (WohlfartKugelberg Welander disease) is the milder form of SMA with onset usually after 18 months of age. ${ }^{8}$ Study reported $>95 \%$ children with SMA have homozygous deletion of Survival Motor Neuron1 (SMN1) gene and other patients may have changes in SMN2 gene. ${ }^{9}$

This results in reduced level of SMN protein leading to degeneration of alpha motor neuron of spinal cord. ${ }^{1}$ The highly homologous copy gene (SMN2) is retained in almost all patient but fails to generate adequate SMN protein due to defective splicing pattern. The severity of SMA phenotype is inversely related with SMN2 copy number. ${ }^{9}$ At least 1 copy of SMN 2 is required for development of SMA and type 3 patients have 3-4 copies of SMN2 genes, so it is less severe type of SMA. ${ }^{10}$

SMA type 3 also called Kugelberg-Welander disease is relatively milder form of SMA characterized by proximal muscle weakness and hypotonia caused by degeneration of lower motor neurons in the spinal cord and brainstem nuclei. The onset of disease usually occurs in childhood after ambulation has been achieved, distinguishing two subtypes of the disease: SMA $3 a$ (onset $<3$ years) and SMA $3 b$ (onset $>3$ years). ${ }^{11}$ Although these patients appear normal during infancy, there is a slow but progressive weakness of the limbs and bulbar dysfunction may occur late in the disease. ${ }^{3}$ Type 3 SMA patients usually presents with symptoms of frequent fall, difficulty in climbing stairs and features of proximal muscle weakness. ${ }^{12}$ The reported patient was normal during infancy, achieved all motor skills but never learnt to sit or stand without any assistance. He also had history of frequent fall and gradually developed features of proximal muscle weakness as he had difficulty in combing hair and raising hands above head. The characteristics clinical signs of SMA includes hypotonia, paresis and areflexia. ${ }^{1}$ The child had hypotonia along with absent jerks in all four limbs. In a previous case report along with proximal muscle weakness they also found worm like fasciculation mostly in tongue and both lower limbs. ${ }^{13}$ In this child there was no fasciculation. About $50 \%$ children with type 3 SMA patients loss their ability to walk independently by age of 14 years and some children develop scoliosis and joint contractures. ${ }^{4}$ The present case is quite young in that respect.

The creatine kinase (CK), remain normal in SMA type 1 but may slightly raised in other types of SMA. ${ }^{4}$ In present case muscle enzymes were normal. The SMA patients also have electrophysiological evidence of denervation with slowing of motor conduction velocity, while conduction is found intact in motor and sensory nerves. ${ }^{15}$ In reported patient the motor and sensory nerve conduction velocity were normal, but the Electromyography (EMG) revealed low amplitude and shorter duration of motor conduction velocity mostly in the proximal muscles (biceps brachii, tibialis anterior, quadriceps and gluteus medius). The motor nerve conduction velocity is generally considered normal in diseases of anterior horn cells, including spinal muscular atrophy as the myelin sheath of peripheral nerves are normal. ${ }^{16}$

Muscle biopsy which is an invasive procedure cannot distinguish clearly between SMA subtypes but certain histopathological features are associated with disease severity. ${ }^{13}$ Muscle biopsy in type 1 and 2 shows large group of atrophic fibers interspersed with fascicles of hypertrophied and normal fibers. ${ }^{17}$ In type 3 SMA there are typically group of uniformly atrophic fibers between groups of non atrophic fibers. ${ }^{13}$ 
Muscle biopsy in this patient showed small diameter muscle fibers with mild stromal fatty infiltration compatible with spinal muscular atrophy type 3 . The genetic testing is considered as gold standard. ${ }^{13}$ to confirm the diagnosis which was not done due to lack of facility in the country.

Two differential diagnoses were considered. First one was congenital myopathy. Patients with congenital myopathy usually present muscle weakness since birth, has facial dysmorphism, ptosis, predominantly axial hypotonia, severe respiratory involvement since birth. ${ }^{18}$ These features did not mach with the index patient. Also they have typical findings in muscle biopsy specific for different types of congenital myopathy but present patient had findings compatible with SMA on muscle biopsy. Another differential was Duchenne muscular dystrophy where the boy presents with motor developmental delay, calf muscle hypertrophy, joint contractures, progressive muscle weakness, cognitive impairment and features of cardiomyopathy, with markedly elevated serum Creatine kinase (CK) ${ }^{19}$ These findings also did not match with this patient.

Until last year no treatment was available for SMA and management consisted of supportive measures. Currently a new therapeutic option became available. Nusinersen is the first approved drug to treat pediatric and adult patient with SMA. ${ }^{20}$ Nusinersen is an antisense oligonucleotide that is designed to increase the expression of survival motor neuron protein. It is administered by intrathecal injection for four loading dose with first three loading dose at 14 days interval and fourth dose is given 30 days after third dose. Thereafter maintenance dose is given once every month. ${ }^{20}$ As this drug is still not available, the child was not given the advise. In addition to drug therapy gene therapy approaches using viral vectors to replace SMN1 have been evaluated for SMA. ${ }^{21}$ Stem cell approaches offer promise as a cellular replacement strategy in the treatment of SMA and it is currently receiving considerable attention. ${ }^{22}$ These treatment options are also not available in this country. Counseling the parents about the disease and it's prognosis was done with advise for follow up. Considering the high carrier frequency, carrier screening was requested for the siblings of the parents and also of the parents of SMA children who may help in reproductive planning. ${ }^{3}$ Prenatal diagnosis should be offered to couples who had previously had a child affected with SMA (recurrence rate $25 \%) .{ }^{3}$ This patient has an affected sib so carrier screening is required for the parents, but as genetic testing is not available so parents were counseled about risk of recurrence and about prenatal diagnosis in future pregnancy.

The life expectancy of type 3 SMA patients are not significantly less than normal population. ${ }^{10} \mathrm{~A}$ previous study showed that probabilities of being able to walk at 10 years after the onset of disease was $70.3 \%$ for SMA 3a, $96.7 \%$ for SMA 3b and at 40 years it was $22 \%$ for SMA $3 a, 58.7 \%$ for SMA3b. ${ }^{10}$ The index patient was advised for follow up and also the parents were counseled about the complications (progressive walking difficulty, joint contracture, deformity) which may develop later during the course of illness.

\section{Conclusion:}

SMA is a genetic disease affecting the part of nervous system that controls the voluntary muscle movement. Among the different types of SMA, SMA type 3 is the late onset relatively milder form and slowly progressive. Though these patients may develop several complications later in the course of illness but life expectancy is usually normal. No medical treatment is available in our country which can delay the progression of the disease. Counseling including genetic counseling is of great importance.

\section{References:}

1. Baioni MTC, Ambiel CR. Spinal muscular atrophy: diagnosis, treatment and future prospects. Journal de Pediatria. 2010; 86: 4.

2. Prior TW. Spinal muscular atrophy diagnostics. J. Child Neurol. 2007; 22: 952-6.

3. Amico AD, Mercuri E, Tiziano FD, Bertini E. Spinal muscular atrophy. Orphanet J. Rare Dis. 2011;6: 71-80.

4. Mecuri E, Bertini E, lannaccone ST. Childhood spinal muscular atrophy: controversies and challenges. Lancet Neurol. 2012; 11: 443-52.

5. Kaufmann P, McDermott MP, Darras BT, Finkel RS, Sproule DM, Kang PB et al. Prospective cohort study of spinal muscular atrophy type 2 and 3. Neurology. 2012; 79: 1889-97.

6. Rassman BS. Spinal muscular atrophy: clinical classification and disease heterogenicity. J. Child Neurol. 2007; 22: 946-51.

7. Kolb SJ, Kissel JT. Spinal Muscular Atrophy: A timely Review. Arch neurol. 2011; 68: 979-81. 
8. Biros I, Forrest S. Spinal muscular atrophy: untangling the knot? Genetic neuromuscular disoreders.2014; 68:303-5.

9. Kuzma-Kozakiewicz M, Jedrzejowska M, Kazmierczak B. SMN1 gene duplications are more frequent in progressive muscular atrophy. Amyotroph Lateral Scler Frontotemporal Degner. 2013; 14: 457-62.

10. Forrest E, Lusakowska A, Borkowska J, Petrusewicz IH. A collaborative study on the natural history of childhood and juvenile onset proximal spinal muscular atrophy (type 2 and 3 SMA): 569 patients. Journal of the neurological sciences. 1997; 146: 67-72.

11. Angelini C. Spinal Muscular Atrophy Type3, Kugelberg-Welander disease. Genetic neuromuscular disorders.2014; 68:303-5.

12. Arnold WD, Kassar D, Kissel JT. Spinal Muscular Atrophy: Diagnosis and Management in a New Therapeutic Era. Muscle Nerve. 2015; 51: 157-67.

13. Mustaq F, Ahmed SM, Kaur S, Singh S, Dashora LS. Spinal muscular atrophy. International Journal of Clinical and Experimental Physiology. 2016;3:147-8.

14. Ashrafzadeh F, Nabavi AS, Asadian N, Akhondian J, Toosi MB. Spinal Muscular Atrophy: A short review article. International Journal of Paediatrics. 2014;2:211-5.
15. Wirth B, Brichta L, Hehnen E. Spinal muscular atrophy: from gene to therapy. Seminpediatr neurol.2006;13:121-31.

16. Munsat, TL, Woods R, Fowler W, Pearson CM. Neurogenic muscular atrophy of infancy with prolonged survival.Brain.1969; 92: 9.

17. Fenichel GM, Engel WK. Histochemistry of muscle in Spinal Muscular Atrophy. Neurology.1963;13:1059-66.

18. Kathryn N. Approach to the diagnosis of congenital myopathies. Neuromuscular disorders. 2014; 24:97-116.

19. Jean K Mah. Current and emerging treatment strategies for Duchenne muscular dystrophy. Neuropsychiatric Dis Treat. 2016;12: 17951807.

20. Tabet R, Bitar SE, Zaidan J, Dabaghian G. Spinal Muscular Atrophy: The Treatment Approved. Cureus. 2017;9: 44.

21. Passini MA, Bu J, Roskelley EM, Richards AM, Sardi SP, Klinger KW et al. CNS targeted gene therapy improves survival and motor function in a mouse model of spinal muscular atrophy. $\mathrm{J}$ Clininvest. 2010; 120:1253-64.

22. Harper JM, Krishna C, Darman JS, Deshpande DM, Peck S, Shats I et al. Axonal growth of embryonic stem cell derived motor neuroninjured adult rats. ProcNatlAcadSci USA. 2004; 101:7123-8. 